\title{
Upper critical field of a $D$-wave superconductor stabilized by antiferromagnetic fluctuations
}

\author{
M PROHAMMER and J P CARBOTTE \\ Physics Department, McMaster University Hamilton, Ontario, Canada L8S 4M1
}

\begin{abstract}
We have derived microscopic equations for the upper critical magnetic field of a $D$ wave superconductor stabilized by antiferromagnetic spin fluctuations. We present numerical results for the reduced field $h_{c 2}(t)$ as a function of reduced temperature $t$ when the magnetic field is along the $z$ axis and also zero-order results when it is along the $x$ or $y$ axis. Two different $D$-wave models are considered. The angular dependence of $H_{c 2}$ for $T$ near $T_{c}$ is given as a function of polar angle $\theta$ in the $z-x$ or $z-y$ plane.
\end{abstract}

Keywords. Upper critical field; $D$-wave superconductor; anisotropic superconductivity.

\section{Introduction}

Several authors have studied the possibility of $D$-wave superconductivity stabilized through antiferromagnetic spin fluctuations (Miyake 1987; Miyake et al 1984, 1986; Millis et al 1988; Williams and Carbotte 1989). The model could apply to the heavy fermion superconductors (Steglich 1985; Rauchschwalbe et al 1987) and possibly to other materials. Scharnberg and Klemm $(1980,1981,1985)$ calculated the upper critical magnetic field $H_{c 2}(T)$ for anisotropic superconductors with particular emphasis on the $P$-wave case which implies triplet spin pairing. Here we consider $D$-wave pairing with singlet spin.

\section{Formalism}

The interelectron interaction for scattering from $\mathbf{K}$ to $\mathbf{K}^{\prime}$ on the Fermi surface is given in terms of the electron spin interaction $I$ and the magnetic susceptibility $\chi\left(\mathbf{q}, \omega_{n}\right)$ with $\omega_{n}$ the $n^{\prime}$ th Matsubara frequency. Using a separable model, we write for this interaction

$$
I^{2} \chi\left(\mathbf{K}_{F}-\mathbf{K}_{F}^{\prime} ; \omega_{n}-\omega_{m}\right) \equiv I^{2} \chi_{0}\left(\mathbf{K}_{F}-K_{F}^{\prime}\right) \boldsymbol{\Phi}\left(\omega_{n}-\omega_{m}\right),
$$

with $\chi_{0}$ the static susceptibility. The spectral representation for $\Phi$ is

$$
\Phi\left(\omega_{n}-\omega_{m}\right)=\frac{2}{I^{2} N(0) \pi} \int_{0}^{x} \mathrm{~d} \omega \frac{\omega A_{\mathrm{SF}}(\omega)}{\omega^{2}+\left(\omega_{n}-\omega_{m}\right)^{2}}
$$

with $N(0)$ the electron density of states at the Fermi surface and $A_{\mathrm{SF}}(\omega)$ a spectral density for spin fluctuations.

A simple model for $\chi_{0}$ is

$$
I^{2} \chi_{0}\left(\mathbf{K}_{\boldsymbol{F}}^{\prime}-\mathbf{K}_{\boldsymbol{F}}\right)=J_{0}-J_{1} \eta_{i}(\hat{\mathbf{K}}) \eta_{i}\left(\hat{\mathbf{K}}^{\prime}\right)
$$

where

$$
\eta_{1}(\hat{\mathbf{K}})=\frac{\sqrt{15}}{2}\left(\hat{k}_{x}^{2}=\hat{k}_{y}^{2}\right) \quad \text { and } \quad \eta_{2}(\hat{\mathbf{K}})=\frac{\sqrt{5}}{2}\left(\hat{K}_{x}^{2}+\hat{K}_{y}^{2}-2 \hat{K}_{z}^{2}\right)
$$


The ratio of $J_{1}$ to $J_{0}$ is denoted by $g$ and the equation for the space $(\mathbf{R})$ momentum $(\mathbf{K})$ and energy $\left(\omega_{n}\right)$-dependent gap $\phi\left(\mathbf{R}, \mathbf{K}, \omega_{n}\right)$ which we assume to be of the form $\phi(R) \Delta\left(\omega_{n}\right) \eta_{i}(\hat{\mathbf{K}})$ is

$$
\begin{aligned}
\phi(\mathbf{R}) \Delta\left(\omega_{n}\right)= & \frac{g T}{2 v_{F}} \sum_{m} \int \frac{\mathrm{d}^{3} K}{K^{2}} \eta_{i}^{2}(\hat{\mathbf{K}}) \exp \left[-\left(2\left|\omega_{m}\right| K\right) / v_{F}\right] \\
& \exp \left[-i \operatorname{sgn}\left(\omega_{m}\right) \mathbf{K} \pi(R)\right] \times \lambda\left(\omega_{n}-\omega_{m}\right) \phi(\mathbf{R}) \Delta\left(\omega_{m}\right) .
\end{aligned}
$$

Here $T$ is the temperature and $v_{F}$ the Fermi velocity. The operator $\pi(R)=1 / i \nabla_{\mathbf{R}}$ $+2 e A(\mathbf{R})$ where $e$ is the electron charge and $\mathbf{A}$ is the vector potential. Finally

$$
\lambda\left(\omega_{n}-\omega_{m}\right)=\int_{0}^{\infty} \mathrm{d} \omega \frac{2 \omega A_{\mathrm{SF}}(\omega)}{\omega^{2}+\left(\omega_{n}-\omega_{m}\right)^{2}} .
$$

To solve (5), it is necessary, in general, to expand $\phi(\mathbf{R})$ in a complete set of functions. We can use the generalized Abrikosov solutions $\phi_{N}(\mathbf{R})$ (Scharnberg and Klemm 1980, 1981, 1985) and write

$$
\phi(\mathbf{R})=\sum_{N=0}^{\infty} b_{N} \phi_{N}(\mathbf{R})
$$

\section{Results}

For the magnetic field $H$ along the $z$-axis the lowest Abrikosov solution $\phi_{0}$ is the complete solution for the $\eta_{2}(\hat{\mathbf{K}})$ model. Results for the reduced upper critical field $h_{c 2}(t)$ as a function of reduced temperature are shown as the short dashed line in the upper
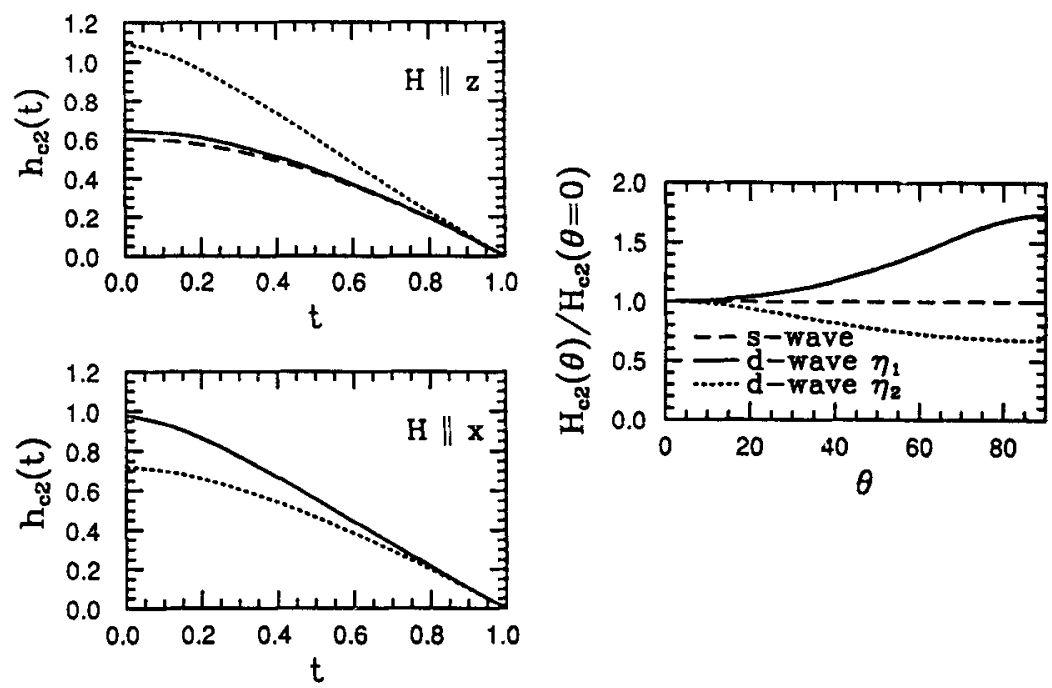

Figure 1. Reduced critical field $h_{c 2}(t)$ as a function of reduced temperature $t$. The top left frame is for the magnetic field $H$ parallel to the $z$ axis. The short-dashed curve applies to the $\eta_{2}$ model and represents a complete solution. The solid and long dashed curve apply to the $\eta_{1}$ model with $N=0$ (long dashed) and $N=0,4$ (solid) solutions respectively. The lower left frame holds for $H$ parallel to the $x$ or $y$ axis and gives approximate results only with $N=0$. The right frame gives $H_{c 2}(\theta) / H_{c 2}(\theta=0)$ near $T_{c}$ as a function of angle $\theta$ in the $z-x$ or $z-y$ plane with $\theta=0$ to the $z$ axis. 
frame left of figure 1 . Note the upward curvature near $t=1$. The $t=0$ value is much higher than for an $S$-wave superconductor $\left(h_{c 2}(0)=0.73\right)$ and the $p$-wave polar state $\left(h_{\mathrm{c} 2}(0)=0.85\right)$. The case applies for $g=1$ and $T_{c} / \omega_{\mathrm{SF}}=0.05$ with $\omega_{\mathrm{SF}}$ the spin fluctuation frequency in the spectral density $A_{\mathrm{SF}}(\omega)$ which is taken to be a delta function. The corresponding value of $\lambda(0)=0.56$. To get a complete solution for the $\eta_{1}(\hat{\mathbf{K}})$ model, one needs $N=0,4,8,12 \ldots$ etc. Results with $N=0$ only are given as the long dashed line in the top left frame of figure 1 . We see that the temperature dependence of $h_{c 2}(t)$ is now completely different from that found in the $\eta_{2}(\hat{\mathbf{K}})$ model and is closer to the familiar $S$-wave dependence. Adding on the $N=4$ function in the expansion for $\phi(\mathbf{R})$ gives the solid curve which is everywhere higher than the long dashed curve. The curves differ most at $t=0$, although the differences are not very large, indicating that an expansion in $N$ retaining only the two lowest terms is probably fairly accurate.

In the lower left frame of figure 1, we show results for $h_{c 2}(t)$ vs $t$ when $H$ is parallel to the $x$ or $y$ axis. In this case, $\phi_{N}$ 's with $N=0,2,4,6,8 \ldots$, all contribute to $h_{c 2}(t)$. We present results only for $N=0$. In contrast to the previous case $(H \| z)$, the solid curve for $\eta_{1}(\hat{\mathbf{K}})$ is now higher than the short dashed curve for the $\eta_{2}(\hat{\mathbf{K}})$ model. Again, we note that $h_{c 2}(0)$ is quite different from the $S$-wave case (Helfand and Werthamer 1966; Werthamer et al 1966).

Near $T=T_{c}$, we can work out an explicit and simplified equation for $H_{c 2}$ as a function of angle $\theta$ in the $z-x$ or $z-y$ plane. In this case, the Abrikosov solution with $N=0$ is valid and $H_{\mathrm{c} 2}$ is given by the equation

$$
\begin{aligned}
\Delta\left(\omega_{n}\right)= & \mathrm{g} \pi T \sum_{m} \lambda(n-m) \Delta\left(\omega_{m}\right) \\
& \times\left[\frac{1}{\left|\omega_{m}\right|}-\frac{3}{7\left|\omega_{m}\right|^{3}}\left[\frac{\alpha^{*}}{3} \sin ^{2} \theta+\cos ^{2} \theta\right]^{1 / 2}\right] \text { for } \eta_{1}
\end{aligned}
$$

and a similar expression for $\eta_{2}$ with $\frac{3}{7} \rightarrow \frac{3}{21}$ and $\frac{1}{3} \rightarrow \frac{11}{5}$. Here $\alpha^{*}=e H_{c 2} v_{F}^{2} / 2$. Results for $H_{c 2}(\theta) / H_{c 2}(\theta=0)$ are shown in the right frame of figure 1. For an $S$-wave superconductor (long dashed curve), there is no $\theta$ dependence, and the curve is flat at value 1 . For the $\eta_{1}(\hat{\mathbf{K}})$ (solid curve) model, the field increases with $\theta$; while for model $\eta_{2}(\hat{\mathbf{K}})$ (short-dashed curve), it decreases. This result is in accord with the results given in the two upper frames.

The deduced shapes of $h_{c 2}(t)$ for $H \| x$ and $T$ near $T_{c}$ are slightly higher than the ones in the lower left frame of figure 1, indicating that the full results for $h_{c 2}(t)$ will also be higher than the approximate results presented.

\section{Conclusion}

In conclusion, the upper critical field of a $D$-wave superconductor has been calculated for $H \| z$ and $\| x$ or $y$ axis. The dependence on angle is very strong and the dependence on reduced temperature can be very different from the conventional $S$-wave case.

\section{References}


Miyake K 1987 J. Magn. Magn. Mater. 63 \& 64411

Miyake K, Matsuura T, Jichu H and Nagaoka Y 1984 Progr. Theor. Phys. 721063

Miyake K, Schmitt-Rink S and Varma C M 1986 Phys. Rev. B34 6554

Rauchschwalbe U, Ahlheim U, Bredl C D, Mayer H M and Steglich F 1987 J. Magn. Magn. Mater. 63 \& 64 447

Steglich F 1985 in Theory of heavy fermions and valence fluctuations (ed.) P Fulde (New York: Springer) p. 23

Scharnberg K and Klemm R A 1980 Phys. Rev. B22 5233

Scharnberg K and Klemm R A 1981 Phys. Rev. B24 6361

Scharnberg K and Klemm R A 1985 Phys. Rev. Lett. 542445

Werthamer N R, Helfand E and Hohenberg P C 1966 Phys. Ret. 147295

Williams P J and Carbotte J P 1989 Phys. Rev. B3 2180 